\title{
Axions, Majorana neutrino masses and implications for the dark sector of the Universe
}

\author{
Nick E. Mavromatos*i \\ King's College London, Physics Department, Theoretical Particle Physics and Cosmology \\ Group, Strand, London WC2R 2LS, UK \\ E-mail: Nikolaos. Mavromatos@cern.ch
}

\begin{abstract}
We discuss a novel mechanism for generating masses for right-handed Majorana neutrinos, that goes beyond the conventional seesaw. The mechanism involves quantum fluctuations of a massless Kalb-Ramond (KR) pseudoscalar (axion-like) field, which exists in string-inspired extensions of the standard model. We assume a kinetic mixing of the KR field with ordinary (massive in general) axions, which also exist in string models, and which are assumed to couple to Majorana (right-handed) neutrinos via non-perturbatively-generated chirality changing Yukawa couplings, breaking the axion-shift symmetry. No vacuum expectation value is assumed for the axion fields. Majorana masses for the right-handed neutrinos are generated radiatively as a result of anomalous higher-loop axion-neutrino couplings. Implications for the Dark sector of the Universe are discussed. In particular, we explore the possibility of generating masses for the right-handed neutrinos of order of a few tens of $\mathrm{keV}$. Such neutrinos could play an important rôle in the galactic structure, and more generally they could serve as a warm dark matter component in the Universe, providing a potential resolution to the so-called small-scale cosmology "crisis", that is, discrepancies between observations at galactic scales and numerical simulations based on the $\Lambda \mathrm{CDM}$ model. If such scenarios are realised in nature, they might imply that Dark Matter consists of more than one species (warm and cold), with distinct rôles in the structure and evolution of the Universe: the cold one being still responsible for the large scale structure of the Universe, in accordance to the predictions of the $\Lambda \mathrm{CDM}$ model, which agree with a plethora of cosmological observations, but the warm component ( $\mathrm{keV}$ sterile neutrino) playing a crucial rôle in the (observed) galactic structure.
\end{abstract}

Corfu Summer Institute 2017 'School and Workshops on Elementary Particle Physics and Gravity' 2-28 September 2017

Corfu, Greece

\footnotetext{
*Speaker.

${ }^{\dagger}$ This work is supported in part by STFC (UK) under the research grant ST/P000258/1
} 


\section{Introduction and Summary}

The discovery [1] of the Higgs boson at the CERN Large Hadron Collider (LHC) in 2012 constitutes an important milestone for the Ultra-Violet (UV) completion of the Standard Model (SM). Although the so-called Higgs mechanism may well explain the generation of most of the particle masses in the SM, the origin of the small neutrino masses still remains an open issue. In particular, the observed smallness of the light neutrino masses may naturally be explained through the see-saw mechanism [2], which necessitates the Majorana nature of the light (active) neutrinos and postulates the presence of heavy right-handed Majorana partners of mass $M_{R}$. The right-handed Majorana mass $M_{R}$ is usually considered to be much larger than the lepton or quark masses. The origin of $M_{R}$ has been the topic of several extensions of the SM in the literature, within the framework of quantum field theory [2,3] and string theory [4]. However, up to now, there is no experimental evidence for right-handed neutrinos or for any extension of SM, as a matter of fact, although some optimism of discovering supersymmetry in the next round of LHC (operating at $14 \mathrm{TeV}$ energies) exists among particle physicists.

Until therefore such extensions of the SM are discovered, it is legitimate to search for alternative mechanisms for neutrino mass generation, that keep the spectrum of SM intact, except perhaps for the existence of right handed neutrinos that are allowed. Such minimal, non supersymmetric extensions of the Standard Model with three in fact right-handed Majorana neutrinos complementing the three active left-handed neutrinos (termed $v \mathrm{MSM}$ ), have been proposed [5], in a way consistent with current cosmology. Such models are characterised by relatively light right-handed neutrinos, two of which are almost degenerate, with masses of order $\mathrm{GeV}$, and a much lighter one, almost decoupled, with masses in the $\mathrm{keV}$ range, which may play the role of warm dark matter. The $\mathrm{keV}$ neutrino warm dark matter has been argued [6] to play an important rôle in the galactic structure, and more general in providing a resolution to the so-called "small-scale cosmology crisis", that is discrepancies between observations and $\Lambda \mathrm{CDM}$-model-based simulations for the dark matter distribution in galaxies. The right-handed neutrinos serve the purpose of generating, through seesaw type mechanisms, the active neutrino mass spectrum, consistent with observed flavour oscillations. However, there are no suggestions for microscopic mechanisms for the generation of the righthanded neutrino mass spectrum in such scenarios.

Motivated by these facts we review in the next section 2, an alternative proposal for righthanded Majorana neutrino mass generation [7], through the anomalous interaction of these neutrinos with axion-like field that exist in string-inspired extensions of the standard model. These models contain (in their massless gravitational multiplet) a spin-one antisymmetric tensor (KalbRamond) field, and there is an abelian gauge invariance that implies the presence of the latter only through its (three-rank) covariant, totally antisymmetry, tensor field strength $H_{\mu v \rho}$, which in terms of the (lowest order in derivatives - we restrict ourselves to) string effective action appear as a totally antisymmetric part of a torsion. The latter couples, via the gravitational covariant derivative, to the axial fermion current, summed up over all fermion species in the model, including righthanded neutrinos. The generation of (right-handed, sterile) neutrino masses in that case proceeds, as we shall review below, via chiral anomalous three-loop graphs of neutrinos interacting with the totally antisymmetric torsion quantum KR field. In four space-time dimensions, the latter is represented as an axion field, whose (kinetic) mixing with ordinary axion fields, that in turn interact 
with the Majorana right-handed neutrinos via chirality changing Yukawa couplings, is held responsible for the right-handed Majorana neutrino mass generation. We discuss specifically under which circumstances low (in the keV range) masses for the lightest of the sterile right-handed neutrinos can be generated, which may have important implications for the (warm) dark matter sector of the Universe. These are discussed in section 3, where it is argued that sterile neutrinos with masses of a few tens of $\mathrm{keV}$ can play an important rôle in providing agreement between theory and observations for the galactic structure, and more generally for alleviating some of the tensions of the so-called "small-scale" cosmology "crisis" [6]. Our conclusions are presented in section 4.

\section{Axions and radiatively generated Majorana neutrino masses}

Let us commence our discussion by considering first a rather generic discussion concerning the propagation of Dirac fermions in a torsionful space-time. The extension to the Majorana case is straightforward. We shall restrict ourselves to the specific case of interest to us here, in which the (totally antisymmetric) torsion is provided by the antisymmetric tensor Kalb-Ramond field later on.

The relevant action reads:

$$
S_{\psi}=\frac{i}{2} \int d^{4} x \sqrt{-g}\left(\bar{\psi} \gamma^{\mu} \overline{\mathscr{D}}_{\mu} \psi-\left(\overline{\mathscr{D}}_{\mu} \bar{\psi}\right) \gamma^{\mu} \psi\right)
$$

where $\overline{\mathscr{D}}_{\mu}=\bar{\nabla}_{\mu}+\ldots$, is the covariant derivative (including gravitational and gauge-field connection parts, in case the fermions are charged). The overline above the covariant derivative, i.e. $\bar{\nabla}_{\mu}$, denotes the presence of torsion, which is introduced through the torsionful spin connection: $\bar{\omega}_{a b \mu}=\omega_{a b \mu}+K_{a b \mu}$, where $K_{a b \mu}$ is the contorsion tensor. The latter is related to the torsion two-form $\mathbf{T}^{a}=\mathbf{d} \mathbf{e}^{a}+\bar{\omega}^{a} \wedge \mathbf{e}^{b}$ via [8,9]: $K_{a b c}=\frac{1}{2}\left(\mathrm{~T}_{c a b}-\mathrm{T}_{a b c}-\mathrm{T}_{b c d}\right)$. The presence of torsion in the covariant derivative in the action (2.1) leads, apart from the standard terms in manifolds without torsion, to an additional term involving the axial current

$$
J_{5}^{\mu} \equiv \bar{\psi} \gamma^{\mu} \gamma^{5} \psi
$$

The relevant part of the action reads:

$$
S_{\psi} \ni-\frac{3}{4} \int d^{4} \sqrt{-g} S_{\mu} \bar{\psi} \gamma^{\mu} \gamma^{5} \psi=-\frac{3}{4} \int S \wedge^{\star} J^{5}
$$

where $\mathbf{S}={ }^{\star} \mathbf{T}$ is the dual of $\mathbf{T}: S_{d}=\frac{1}{3 !} \varepsilon^{a b c}{ }_{d} T_{a b c}$.

We next remark that the torsion tensor can be decomposed into its irreducible parts [8], of which $S_{d}$ is the pseudoscalar axial vector: $T_{\mu v \rho}=\frac{1}{3}\left(T_{v} g_{\mu \rho}-T_{\rho} g_{\mu v}\right)-\frac{1}{3 !} \varepsilon_{\mu v \rho \sigma} S^{\sigma}+q_{\mu v \rho}$, with $\varepsilon_{\mu \nu \rho \sigma} q^{v \rho \sigma}=q_{\rho \nu}^{v}=0$. This implies that the contorsion tensor undergoes the following decomposition:

$$
K_{a b c}=\frac{1}{2} \varepsilon_{a b c d} S^{d}+\widehat{K}_{a b c}
$$

where $\widehat{K}$ includes the trace vector $T_{\mu}$ and the tensor $q_{\mu v \rho}$ parts of the torsion tensor.

The gravitational part of the action can then be written as: $S_{G}=\frac{1}{2 \kappa^{2}} \int d^{4} x \sqrt{-g}(R+\widehat{\Delta})+$ $\frac{3}{4 \kappa^{2}} \int \mathbf{S} \wedge{ }^{\star} \mathbf{S}$, where $\widehat{\Delta}=\widehat{K}_{\mu \nu}^{\lambda} \widehat{K}_{\lambda}^{v \mu}-\widehat{K}^{\mu \nu}{ }_{\nu} \widehat{K}_{\mu \lambda}{ }^{\lambda}$, with the hatted notation defined in (2.4). 
In a quantum gravity setting, where one integrates over all fields, the torsion terms appear as non propagating fields and thus they can be integrated out exactly. The authors of [9] have observed though that the classical equations of motion identify the axial-pseudovector torsion field $S_{\mu}$ with the axial current, since the torsion equation yields

$$
K_{\mu a b}=-\frac{1}{4} e_{\mu}^{c} \varepsilon_{a b c d} \bar{\psi} \gamma_{5} \tilde{\gamma}^{d} \psi
$$

From this it follows $\mathbf{d}^{\star} \mathbf{S}=0$, leading to a conserved "torsion charge" $Q=\int{ }^{\star} \mathbf{S}$. To maintain this conservation in quantum theory, they postulated $\mathbf{d}^{\star} \mathbf{S}=0$ at the quantum level, which can be achieved by the addition of judicious counter terms. This constraint, in a path-integral formulation of quantum gravity, is then implemented via a delta function constraint, $\delta\left(d^{\star} \mathbf{S}\right)$, and the latter via the well-known trick of introducing a Lagrange multiplier field $\Phi(x) \equiv\left(3 / \kappa^{2}\right)^{1 / 2} b(x)$. Hence, the relevant torsion part of the quantum-gravity path integral would include a factor

$$
\begin{aligned}
& \mathscr{Z} \propto \int D \mathbf{S} D b \exp \left[i \int \frac{3}{4 \kappa^{2}} \mathbf{S} \wedge^{\star} \mathbf{S}-\frac{3}{4} \mathbf{S} \wedge^{\star} \mathbf{J}^{5}+\left(\frac{3}{2 \kappa^{2}}\right)^{1 / 2} b d^{\star} \mathbf{S}\right] \\
& =\int D b \exp \left[-i \int \frac{1}{2} \mathbf{d} b \wedge^{\star} \mathbf{d} b+\frac{1}{f_{b}} \mathbf{d} b \wedge^{\star} \mathbf{J}^{5}+\frac{1}{2 f_{b}^{2}} \mathbf{J}^{5} \wedge^{\star} \mathbf{J}^{5}\right]
\end{aligned}
$$

where $f_{b}=\left(3 \kappa^{2} / 8\right)^{-1 / 2}=\frac{M_{P}}{\sqrt{3 \pi}}$ and the non-propagating $\mathbf{S}$ field has been integrated out. The reader should notice that, as a result of this integration, the corresponding effective field theory contains a non-renormalizable repulsive four-fermion axial current-current interaction, characteristic of any torsionful theory [8].

The torsion term, being geometrical, due to gravity, couples universally to all fermion species, not only neutrinos. Thus, in the context of the SM of particle physics, the axial current (2.2) is expressed as a sum over fermion species

$$
J_{5}^{\mu} \equiv \sum_{i=\text { fermion species }} \bar{\psi}_{i} \gamma^{\mu} \gamma^{5} \psi_{i}
$$

In theories with chiral anomalies, like the quantum electrodynamics part of SM, the axial current is not conserved at the quantum level, due to anomalies, but its divergence is obtained by the one-loop result [10]:

$$
\begin{aligned}
\nabla_{\mu} J^{5 \mu} & =\frac{e^{2}}{8 \pi^{2}} F^{\mu v} \widetilde{F}_{\mu v}-\frac{1}{192 \pi^{2}} R^{\mu v \rho \sigma} \widetilde{R}_{\mu v \rho \sigma} \\
& \equiv G(\mathbf{A}, \omega) .
\end{aligned}
$$

We may then partially integrate the second term in the exponent on the right-hand-side of (2.6) and take into account (2.8). The reader should observe that in (2.8) the torsion-free spin connection has been used. This can be achieved by the addition of proper counter terms in the action [9], which can convert the anomaly from the initial $G(\mathbf{A}, \bar{\omega})$ to $G(\mathbf{A}, \omega)$. Using (2.8) in (2.6) one can then obtain for the effective torsion action in theories with chiral anomalies, such as the QED part of the SM:

$$
\int D b \exp \left[-i \int \frac{1}{2} \mathbf{d} b \wedge^{\star} \mathbf{d} b-\frac{1}{f_{b}} b G(\mathbf{A}, \omega)+\frac{1}{2 f_{b}^{2}} \mathbf{J}^{5} \wedge^{\star} \mathbf{J}^{5}\right]
$$


A concrete example of torsion is provided by string-inspired theories, where the totally antisymmetric component $S_{\mu}$ of the torsion is identified with the field strength of the spin-one antisymmetric tensor (Kalb-Ramond (KR) [11]) field $H_{\mu v \rho}=\partial_{[\mu} B_{v \rho]}$, where the symbol [...] denotes antisymmetrization of the appropriate indices. The string theory effective action depends only on $H_{\mu v \rho}$ as a consequence of the "gauge symmetry" $B_{\mu v} \rightarrow B_{\mu v}+\partial_{[\mu} \Theta_{v]}$ that characterises all string theories. It can be shown [12] that the terms of the effective action up to and including quadratic order in the Regge slope parameter $\alpha^{\prime}$, of relevance to the low-energy (field-theory) limit of string theory, which involve the $\mathrm{H}$-field strength, can be assembled in such a way that only torsionful Christoffel symbols, $\bar{\Gamma}_{v \rho}^{\mu}$ appear: $\bar{\Gamma}_{v \rho}^{\mu}=\Gamma_{v \rho}^{\mu}+\frac{\kappa}{\sqrt{3}} H_{v \rho}^{\mu} \neq \bar{\Gamma}_{\rho v}^{\mu}$, where $\Gamma_{v \rho}^{\mu}=\Gamma_{\rho v}^{\mu}$ is the ordinary, torsion-free, symmetric connection, and $\kappa$ is the gravitational constant. In four space-time dimensions, the dual of the $\mathrm{H}$-field is indeed the derivative of an (KR) axion-like field, analogous to the field $b$ above, $H_{\mu v \rho} \propto \varepsilon_{\mu v \rho \sigma} \partial^{\sigma} b(x)$. For completeness we mention at this point that background geometries with (approximately) constant background $H_{i j k}$ torsion (corresponding to a linear in cosmic time KR axion), where Latin indices denote spatial components of the four-dimensional space-time, may characterise the early universe. In such cases, the H-torsion background constitutes extra source of $\mathrm{CP}$ violation, necessary for lepotogenesis, and through Baryon-minus-Lepton-number (B-L) conserving processes, Baryogenesis, and thus the observed matter-antimatter asymmetry in the Universe [13]. Today of course any torsion background should be strongly suppressed, due to the lack of any experimental evidence for it [14]. Scenarios as to how such cosmologies can evolve so as to guarantee the absence of any appreciable traces of torsion today can be found in [13].

In what follows we shall consider the effects of the quantum fluctuations of such a $\mathrm{KR} \mathrm{H}$ torsion, which survive the absence of any torsion background. An important aspect of the coupling of the $H$-torsion (or, equivalently, the KR axion quantum field $b(x)$ ) to the fermionic matter discussed above is its shift symmetry, characteristic of an axion field. Indeed, by shifting the field $b(x)$ by a constant: $b(x) \rightarrow b(x)+c$, the action (2.9) only changes by total derivative terms, such as $c R^{\mu v \rho \sigma} \widetilde{R}_{\mu \nu \rho \sigma}$ and $c F^{\mu v} \widetilde{F}_{\mu v}$. These terms are irrelevant for the equations of motion and the induced quantum dynamics, provided the fields fall off sufficiently fast to zero at space-time infinity. The scenario for the anomalous Majorana mass generation through torsion proposed in [7], and reviewed here, consists of augmenting the effective action (2.9) by terms that break such a shift symmetry. To illustrate this last point, we first couple the KR axion $b(x)$ to another pseudoscalar axion field $a(x)$. In string-inspired models, such pseudoscalar axion $a(x)$ may be provided by the string moduli [15]. The proposed coupling occurs through a mixing in the kinetic terms of the two fields. To be specific, we consider the action (henceforth we restrict ourselves to right-handed Majorana neutrino fermion fields):

$$
\begin{aligned}
\mathscr{S}= & \int d^{4} x \sqrt{-g}\left[\frac{1}{2}\left(\partial_{\mu} b\right)^{2}+\frac{b(x)}{192 \pi^{2} f_{b}} R^{\mu v \rho \sigma} \widetilde{R}_{\mu v \rho \sigma}+\frac{1}{2 f_{b}^{2}} J_{\mu}^{5} J^{5^{\mu}}+\gamma\left(\partial_{\mu} b\right)\left(\partial^{\mu} a\right)+\frac{1}{2}\left(\partial_{\mu} a\right)^{2}\right. \\
& \left.-y_{a} i a\left(\bar{\psi}_{R}^{C} \psi_{R}-\bar{\psi}_{R} \psi_{R}^{C}\right)\right]+\ldots,
\end{aligned}
$$

where the ... indicate terms in the low-energy string effective action, including SM ones, that are not of direct relevance to our purposes in the present article. Above, $\psi_{R}^{C}=\left(\psi_{R}\right)^{C}$ is the chargeconjugate right-handed fermion $\psi_{R}, J_{\mu}^{5}=\bar{\psi} \gamma_{\mu} \gamma_{5} \psi$ is the axial current of the four-component Majorana fermion $\psi=\psi_{R}+\left(\psi_{R}\right)^{C}$, and $\gamma$ is a real parameter to be constrained later on. Here, we have ignored gauge fields, which are not of interest to us, and the possibility of a non-perturbative 


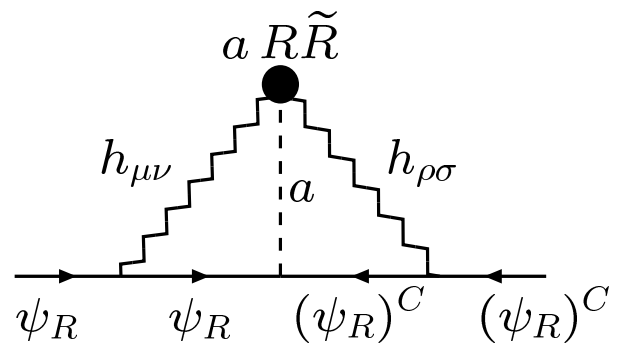

Figure 1: Typical higher-loop Feynman graph giving rise to anomalous fermion mass generation [7]. The black circle denotes the operator $a(x) R_{\mu \nu \lambda \rho} \widetilde{R}^{\mu v \lambda \rho}$ induced by KR torsion. The fields $h_{\mu v}$ (wavy lines) denote graviton fluctuations. Straight lines with arrows denote right handed neutrino fields and their conjugates.

mass $M_{a}$ for the pseudoscalar field $a(x)$. Moreover, we remind the reader that the repulsive selfinteraction fermion terms are due to the existence of torsion in the Einstein-Cartan theory. The Yukawa coupling $y_{a}$ of the axion moduli field $a$ to right-handed sterile neutrino matter $\psi_{R}$ may be due to non perturbative effects. These terms break the shift symmetry: $a \rightarrow a+c$.

It is convenient to diagonalize the axion kinetic terms by redefining the KR axion field as follows: $b(x) \rightarrow b^{\prime}(x) \equiv b(x)+\gamma a(x)$. This implies that the effective action (2.10) becomes:

$$
\begin{aligned}
& \mathscr{S}=\int d^{4} x \sqrt{-g}\left[\frac{1}{2}\left(\partial_{\mu} b^{\prime}\right)^{2}+\frac{1}{2}\left(1-\gamma^{2}\right)\left(\partial_{\mu} a\right)^{2}\right. \\
& \left.+\frac{1}{2 f_{b}^{2}} J_{\mu}^{5} J^{5^{\mu}}+\frac{b^{\prime}(x)-\gamma a(x)}{192 \pi^{2} f_{b}} R^{\mu v \rho \sigma} \widetilde{R}_{\mu v \rho \sigma}-y_{a} i a\left(\bar{\psi}_{R}^{C} \psi_{R}-\bar{\psi}_{R} \psi_{R}^{C}\right)\right]+\ldots
\end{aligned}
$$

Thus we observe that the $b^{\prime}$ field has decoupled and can be integrated out in the path integral, leaving behind an axion field $a(x)$ coupled both to matter fermions and to the operator $R^{\mu \nu \rho \sigma} \widetilde{R}_{\mu v \rho \sigma}$, thereby playing now the rôle of the torsion field. We observe though that the approach is only valid for $|\gamma|<1$, otherwise the axion field would appear as a ghost, i.e. with the wrong sign of its kinetic terms, which would indicate an instability of the model. This is the only restriction of the parameter $\gamma$. In this case we may redefine the axion field so as to appear with a canonical normalised kinetic term, implying the effective action:

$$
\begin{aligned}
\mathscr{S}_{a} & =\int d^{4} x \sqrt{-g}\left[\frac{1}{2}\left(\partial_{\mu} a\right)^{2}-\frac{\gamma a(x)}{192 \pi^{2} f_{b} \sqrt{1-\gamma^{2}}} R^{\mu \nu \rho \sigma} \widetilde{R}_{\mu \nu \rho \sigma}\right. \\
& \left.-\frac{i y_{a}}{\sqrt{1-\gamma^{2}}} a\left(\bar{\psi}_{R}^{C} \psi_{R}-\bar{\psi}_{R} \psi_{R}^{C}\right)+\frac{1}{2 f_{b}^{2}} J_{\mu}^{5} J^{{ }^{\mu}}\right] .
\end{aligned}
$$

Evidently, the action $\mathscr{S}_{a}$ in (2.12) corresponds to a canonically normalised axion field $a(x)$, coupled both to the curvature of space-time, à la torsion, with a modified coupling $\gamma /\left(192 \pi^{2} f_{b} \sqrt{1-\gamma^{2}}\right)$, and to fermionic matter with chirality-changing Yukawa-like couplings of the form $y_{a} / \sqrt{1-\gamma^{2}}$.

The mechanism for the anomalous Majorana mass generation is shown in Fig. 1. We may now estimate the two-loop Majorana neutrino mass in quantum gravity with an effective UV energy cutoff $\Lambda$. Adopting the effective field-theory framework of [16], the gravitationally induced Majorana mass for right-handed neutrinos, $M_{R}$, is estimated to be:

$$
M_{R} \sim \frac{1}{\left(16 \pi^{2}\right)^{2}} \frac{y_{a} \gamma \kappa^{4} \Lambda^{6}}{192 \pi^{2} f_{b}\left(1-\gamma^{2}\right)}=\frac{\sqrt{3} y_{a} \gamma \kappa^{5} \Lambda^{6}}{49152 \sqrt{8} \pi^{4}\left(1-\gamma^{2}\right)} .
$$


In a UV complete theory such as strings, the cutoff $\Lambda$ and the Planck mass scale $M_{P}$ are related.

In particular, as already mentioned, in string theory there are several axion-like (pseudoscalar) fields $a_{i}(x), i=1,2, \ldots n$, originating from flux fields that exist in the spectrum [15], in addition to the aforementioned $B_{\mu \nu}$ Kalb-Ramond field. One can then assume [7] the existence of Yukawa couplings with right-handed neutrinos, provided some non-perturbative instanton effects are responsible for a breaking of the shift symmetry. These string-theory axion fields could mix with each other. Such a mixing can give rise to reduced UV sensitivity of the two-loop graph shown in Fig. 1. To make this point explicit, let us consider a scenario with $n$ axion fields, $a_{1,2, \ldots, n}$, of which only $a_{1}$ has a kinetic mixing term $\gamma$ with the KR axion $b$ and only $a_{n}$ has a Yukawa coupling $y_{a}$ to right-handed neutrinos $\psi_{R}$. The other axions $a_{2,3, \ldots, n}$ have a next-to-neighbour mixing pattern. In such a model, the kinetic terms of the effective action are given by

$$
\mathscr{S}_{a}^{\text {kin }}=\int d^{4} x \sqrt{-g}\left[\frac{1}{2} \sum_{i=1}^{n}\left(\left(\partial_{\mu} a_{i}\right)^{2}-M_{i}^{2}\right)+\gamma\left(\partial_{\mu} b\right)\left(\partial^{\mu} a_{1}\right)-\frac{1}{2} \sum_{i=1}^{n-1} \delta M_{i, i+1}^{2} a_{i} a_{i+1}\right],
$$

where the mixing mass terms $\delta M_{i, i+1}^{2}$ are constrained to be $\delta M_{i, i+1}^{2}<M_{i} M_{i+1}$, so as to obtain a stable positive mass spectrum for all axions. As a consequence of the next-to-neighbour mixing, the UV behaviour of the off-shell transition $a_{1} \rightarrow a_{n}$, described by the propagator matrix element $\Delta_{a_{1} a_{n}}(p)$, changes drastically, i.e. $\Delta_{a_{1} a_{n}}(p) \propto 1 /\left(p^{2}\right)^{n} \sim 1 / E^{2 n}$. Assuming, for simplicity, that all axion masses and mixings are equal, i.e. $M_{i}^{2}=M_{a}^{2}$ and $\delta M_{i, i+1}^{2}=\delta M_{a}^{2}$, the anomalously generated Majorana mass may be estimated to be

$$
M_{R} \sim \frac{\sqrt{3} y_{a} \gamma \kappa^{5} \Lambda^{6-2 n}\left(\delta M_{a}^{2}\right)^{n}}{49152 \sqrt{8} \pi^{4}\left(1-\gamma^{2}\right)}
$$

for $n \leq 3$, and

$$
M_{R} \sim \frac{\sqrt{3} y_{a} \gamma \kappa^{5}\left(\delta M_{a}^{2}\right)^{3}}{49152 \sqrt{8} \pi^{4}\left(1-\gamma^{2}\right)} \frac{\left(\delta M_{a}^{2}\right)^{n-3}}{\left(M_{a}^{2}\right)^{n-3}},
$$

for $n>3$. It is then not difficult to see that three axions $a_{1,2,3}$ with next-to-neighbour mixing as discussed above would be sufficient to obtain a UV finite (cut-off- $\Lambda$-independent) result for $M_{R}$ at the two-loop level. Of course, beyond the two loops, $M_{R}$ will depend on higher powers of the energy cut-off $\Lambda$, i.e. $\Lambda^{n>6}$, but if $\kappa \Lambda \ll 1$, these higher-order effects are expected to be subdominant.

In the above $n$-axion-mixing scenarios, we note that the anomalously generated Majorana mass term will only depend on the mass-mixing parameters $\delta M_{a}^{2}$ of the axion fields and not on their masses themselves, as long as $n \leq 3$. Instead, for axion-mixing scenarios with $n>3$, the induced Majorana neutrino masses are proportional to the factor $\left(\delta M_{a}^{2} / M_{a}^{2}\right)^{n}$, which gives rise to an additional suppression for heavy axions with masses $M_{a} \gg \delta M_{a}$.

In the multi-axion models outlined above, the anomalously generated Majorana neutrino mass $M_{R}$ will still depend on the Yukawa coupling $y_{a}$ and the torsion-axion kinetic mixing coefficient $\gamma$, besides the assumed UV completion scale $\Lambda$ of quantum gravity. In order to get an estimate of the size of $M_{R}$, we treat the axion masses $M_{a}$ and mass-mixings $\delta M_{a}$ as free parameters to be constrained by phenomenology. Let us assume a $n$-axion-mixing models with $n \geq 3$, in which the axion mass mixing $\delta M_{a}$ and their masses $M_{a}$ are of the same order, i.e. $\delta M_{a} / M_{a} \sim 1$. In this case, employing (2.15), we may estimate the Majorana neutrino mass $M_{R}$ to be

$$
\frac{M_{R}}{M_{a}} \sim \frac{10^{-3} y_{a} \gamma}{1-\gamma^{2}} \frac{\left(\delta M_{a}\right)^{6}}{M_{a} M_{P}^{5}} \sim \frac{10^{-3} y_{a} \gamma}{1-\gamma^{2}}\left(\frac{\delta M_{a}}{M_{P}}\right)^{5} .
$$


For axion masses $M_{a} \leq 1 \mathrm{TeV}$ considered in the literature thus far, we find that $M_{R} / M_{a} \lesssim 10^{-83}$, which implies extra-ordinary small Majorana masses $M_{R}$. An obvious caveat to this result would be to have ultra-heavy axion masses $M_{a}$ close to the GUT scale and/or fine-tune the torsion-axion kinetic mixing parameter $\gamma$ to 1 , in a way such that the factor $\frac{\gamma}{1-\gamma^{2}}$ compensates for the mass suppression $\left(\delta M_{a} / M_{P}\right)^{5}$ in (2.17). The latter possibility, however, might result in an unnaturally large (non-perturbative) "effective" Yukawa coupling $y_{a}^{\text {eff }} \equiv y_{a} / \sqrt{1-\gamma^{2}} \gtrsim \sqrt{4 \pi}$ in (2.12), which will bring us outside the perturbative framework that we have been considering here. A more detailed phenomonological and astrophysical analysis of all possible axion-mixing scenarios falls beyond the scope of the present talk.

\section{Implications for the Dark Sector of the Universe}

It is interesting from a cosmological viewpoint to provide a numerical estimate of the anomalously generated Majorana mass $M_{R}$ given in (2.13) above. Assuming that $\gamma \ll 1$, the size of $M_{R}$ may be estimated from (2.13) to be

$$
M_{R} \sim\left(3.1 \times 10^{11} \mathrm{GeV}\right)\left(\frac{y_{a}}{10^{-3}}\right)\left(\frac{\gamma}{10^{-1}}\right)\left(\frac{\Lambda}{2.4 \times 10^{18} \mathrm{GeV}}\right)^{6} .
$$

Obviously, the generation of $M_{R}$ is highly model dependent. Taking, for example, the quantum gravity scale to be $\Lambda=10^{17} \mathrm{GeV}$, we find that $M_{R}$ is at the TeV scale, for $y_{a}=10^{-3}$ and $\gamma=0.1$. However, if we take the quantum gravity scale to be close to the GUT scale, i.e. $\Lambda=10^{16} \mathrm{GeV}$, we obtain a right-handed neutrino mass $M_{R} \sim 16 \mathrm{keV}$, for the choice $y_{a}=\gamma=10^{-3}$. This is in the preferred ballpark region for the (right-handed) sterile neutrino $\psi_{R}$ to qualify as a warm dark matter (WDM) [17, 18].

Moreover, as discussed in [6], the introduction of appropriate self-interactions among the srerile right-handed neutrino dark matter (DM), can serve as a means of providing an explanation of the observed core-halo galactic structure, upon assuming specific core profiles, specifically, the Ruffini-Argüelles-Rueda profile [19]. In addition, such self-interacting neutrino WDM models can also alleviate some of the discrepancies between numerical simulations based on $\Lambda$ CDM model and observations at galactic scales ("small-scale cosmology crisis"), provided the self interaction total cross section $\sigma_{\text {SIDM }}$ of a dark matter particle with mass $m$ lies in the range

$$
0.1 \leq \frac{\sigma_{\mathrm{SIDM}} / m}{\mathrm{~cm}^{2} g^{-1}} \leq 0.47
$$

where the upper limit has been inferred by recent studies of several merging galaxies, using novel techniques and observables [20].

For completeness, we mention that discrepancies between observations at galactic scales and $\Lambda \mathrm{CDM}$-numerical simulations, appear in three areas:

(i) The Core-Cusp problem (or, as is also known, the cuspy-halo problem), refers to a discrepancy between the observed dark matter density profiles of low-mass galaxies and the density profiles predicted by cosmological N-body simulations. Characteristically, all the $\Lambda$ CDM-based (DM only) simulations form dark matter halos which have "cuspy" dark matter distributions, with the density increasing steeply, i.e. as $\rho \propto r^{-1}$, at small radii. This is, e.g., evidenced in the standard 
Navarro-Frenk-White (NFW) DM profile [21]. On the contrary, the rotation curves of most of the observed dwarf galaxies indicate flat central density profiles ("cores") [22].

(ii) The "missing satellite problem" (or, as is also known, the dwarf galaxy problem), arises from a discrepancy between $\Lambda \mathrm{CDM}$-based numerical cosmological simulations that predict the evolution of the distribution of matter in the universe - pointing towards a hierarchical clustering of DM (where smaller halos merge to form larger halos) - and observations. Although there seem to be enough observed normal-sized galaxies to account for such a numerical distribution, the number of dwarf galaxies is orders of magnitude lower than that expected from the simulations. As a concrete example, we mention that there were observed to be around 38 dwarf galaxies in the Local Group, and only around 11 orbiting the Milky Way, yet one dark matter simulation predicted around 500 Milky Way dwarf satellites [23].

(iii) The too big to fail problem, that is a discrepancy arising between the most massive subhaloes predicted in (dissipationless) $\Lambda \mathrm{CDM}$ simulations and the observed dynamics of the brightest dwarf spheroidal galaxies in the Milky way. In other words, the $\Lambda \mathrm{CDM}$ simulations predict that the most massive subhaloes of the Milky way are too dense to host any of its bright satellites, with luminosity higher than $10^{5}$ the luminosity of the Sun [24].

All three problems have their root in the fact that the cold DM particles, which the $\Lambda$ CDM simulations rely upon, have too short free streaming length during the epochs of galaxy formation, and therefore they form too clumped and too many structures compared to those observed. Although some of these problems could be partly alleviated by more precise observations and/or including baryonic feedback, and may have their root in astrophysical reasons (for instance the missing satellite problem may be attributed partly to the fact that dwarf galaxies have a tendency to merge or may have been stripped apart gravitationally by larger galaxies and hence invisible), nonetheless it would be desirable if a unified explanation is found within the context of fundamental physics, and this is the point of view taken in the approach of including self interactions among the DM particles.

In this latter respect, WDM sterile neutrinos, with masses in the range of tens of $\mathrm{keV}$, as in the $v$ MSM model [5], have been argued to play a rôle, either in the too-big-to-fail problem [25], or, in the self-interacting case [6], for resolving the Core-Cusp problem, because the density profiles based on fermionic phase-space distributions $[19,6]$ develop always an extended plateau on halo scales (starting immediately after the quantum core). The rôle of self interactions (attractive vector interactions due to massive vector fields in the model of [6]) is to make the core more compact than in the non-interacting case [19]. For sterile neutrino masses $m_{\chi}$ in the range [6]

$$
47 \mathrm{keV} \lesssim m_{\chi} \mathrm{c}^{2} \lesssim 345 \mathrm{keV}
$$

where the (robust) upper bound corresponds to the limit of gravitational collapse, the correct DM halo properties of galaxies (in agreement with observations) are guaranteed within the context of the galaxy profile proposed in [19], whilst at the same time the fermi gas of fermionic DM may provide an alternative to the black hole in the centre of the galaxy. It must be stressed though that the lower bound depends crucially on the details of the galactic profile, as well as of the existence (and its detailed properties) of a supermassive black hole in the galactic centre. Hence it is much less robust than the upper bound, and can be further relaxed. 


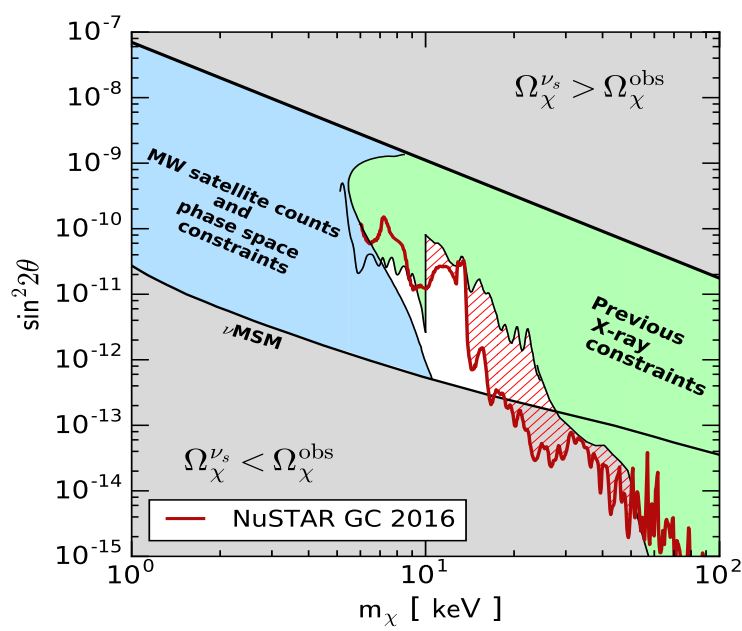

Figure 2: Up-to-date Cosmological and Astrophysical Constraints for the vMSM WDM sterile neutrino mass versus mixing angle with the Standard Model sector (picture from ref. [26]). The recent analysis of Perez et al. [26], employs NuSTAR (Nuclear Spectroscopic Telescope Array) [27] observations of the Galactic Centre, together with additional constraints associated with DM production and its rôle in the structure formation in the Universe, which stem from the requirement that DM comprises predominantly of the lightest of the sterile neutrinos of VMSM. These latest studies exclude almost half of the previously allowed region of VMSM mixing-angle-mass parameters (thick (red) line and hatched region in the figure, the white region being the currently allowed one).

In this respect, we mention a crucial feature of the model of [6]. In that analysis, any mixing of the sterile neutrinos with the Standard Model sector had been ignored, assumed very small. In the spirit of [6], the sterile neutrino might constitute only one component of the DM in the Universe, with other equally dominant DM species in the underlying microscopic model playing different rôles in the structure formation and energy budget of the Cosmos. On the other hand, in the $v$ MSM framework [5], the mixing of the lightest of the sterile right-handed neutrinos which, notably, is assumed to play the rôle of the dominant DM species, having a life-time longer than the Universe age, can be constrained mainly by its decay into X-rays and the DM production and contribution to the large scale structure. The recent constraints of Perez et al. [26], employing observations of the Galactic Centre with the Nuclear Spectroscopic Telescope Array (NuSTAR) [27], when combined with the basic assumption of $v$ MSM [5] that the lightest of the sterile neutrinos provides the dominant component of DM, thus leading to additional constraints for sufficient DM production in the early Universe and efficient rôle in structure formation [17, 18], leaves a very small allowed window in the mixing-angle-sterile-neutrino-mass parameter plane (see white region in fig. 2):

$$
10 \mathrm{keV} \lesssim m_{\chi} \mathrm{c}^{2} \lesssim 16 \mathrm{keV}
$$

As we discussed above ( $c f .(3.1)$ ), within the model of [7], sterile neutrinos can acquire radiatively masses in this range for quite natural values of the parameters.

However, the above region is quite sensitive to the galactic profile used (the authors of [26] used versions of the Navarro-Frenk-White [21] and Einasto [28] profiles in their study). An analysis making use of the Ruffini-Argüelles-Rueda profile [19], employed in the work of [6], may affect the 
conclusions of [26], in particular the upper limit in (3.4). Such a study is still pending. Moreover, the existence of self interactions among the sterile neutrinos in the model of [6], due to a massive vector meson field, will modify significantly the production of the sterile neutrinos as compared with the $v$ MSM model, e.g. sterile neutrinos can he produced in the early Universe by decays of the massive vector field. In the model discussed in the present work [7], there are additional Yukawa interactions of the sterile neutrinos with (massive) axion-like fields, which may well be part of a dark sector of the theory, and there is kinetic mixing of these axions with the KR axion ( $c f$. (2.12),(2.14)). Hence, if the sterile neutrino does not constitute the whole of DM, or its production is modified by additional interactions in a hidden sector of the model, then the lines bounding the gray shaded areas in fig. 2 will be modified. For instance, the lower bound on the mixing angle may be significantly reduced, or even alleviated, if a different mechanism for sterile neutrino production exists. This would imply partial or total relaxation of the associated constraints, in particular as far as the upper bound of (3.4) is concerned. From this general point of view, therefore, diagrams like the one in fig. 2 can only be interpreted as providing upper limits for the mixing parameter of the sterile neutrinos with the Standard Model sector. It goes without saying that the only robust constraints are the ones associated with the maintainance of the predictions of the Big Bag Nucleosynthesis (BBN) mechanism. We hope to come back to a detailed study of such issues within the models of [7] and/or [6] in the near future.

\section{Conclusions and Outlook}

In this talk we reviewed a rather novel mechanism, of semi-geometrical origin, for the generation of sterile right-handed neutrino Majorana masses, which goes beyond the conventional seesaw. The mechanism involves [7] a massless pseudoscalar (axion-like) field (KR axion), which exists in the massless string multiplet of low-energy string-inspired effective field theories. Due to an abelian gauge symmetry of strings, this field appears only in the form of a totally antisymmetric three form $H_{\mu v \rho}$, which, to lowest order in a derivative expansion of the effective low-energy Lagrangian, including gravity, appears as a totally antisymmetric part of a torsion. In four dimensions, $H_{\mu \nu \rho}$ is dual to the aforementioned KR axion. In the model discussed in the talk, the KR axion has a kinetic mixing with other (massive) axions that exist in string-inspired models, which in turn couple with Yukawa-type terms to the right handed neutrinos. Such Yukawa couplings may be generated by non-perturbative string effects that break the shift symmetry that usually characterises axion models.

As a result of such couplings, as well as the torsion-like features of the KR axion field, there is a coupling of quadratic curvature terms to the sterile neutrinos (hence the term "semi-geometrical origin of their mass" used above), through anomalous higher loop graphs, involving axion exchanges (fig. 1). This induces a radiative generation of the sterile (right-handed) neutrino mass, which can be determined in terms of the effective ultraviolet cut-off of the low-energy theory (cf. (3.1)), once the pertinent Yukawa couplings and kinetic mixing parameters of the model are specified (for UV complete theories, like strings, the cut-off scale is related to the string and compactification scales). Upon a rather natural choice of the parameters of the model, the radiatively generated sterile neutrino mass can be made to fit interesting phenomenological models of sterile neutrinos, where the 
lightest of the latter play the rôle of a warm dark matter component, with mass in a few-tens-of-keV range ${ }^{1}$.

In the talk we discussed the pertinent constraints of such models, within our context. We stress once more that our model includes more than one potential species of dark matter: massive string axions and sterile neutrinos, along with other potential DM candidates, originating from an embedding of the model into fully-fledged, phenomenologically realistic, string theories. This feature implies a significant relaxation, in our case, of the constraints characterising other models, like the $v$ MSM, where the sterile neutrinos are considered as the dominant DM species. We have also discussed how (self-interacting) sterile neutrinos with masses of a few tens of $\mathrm{keV}$ can play a rôle in the galactic structure, as well as offer a resolution of some discrepancies between numerical simulations based on $\Lambda \mathrm{CDM}$ model and observations at galactic scales.

I would like to close the talk with the comment that the values of the Yukawa couplings $y_{a}$ appearing in the radiative mass for the sterile neutrinos ((2.13), (2.15) and (2.16)) may be determined by some underlying discrete symmetry [29], which, for instance, allows two of the heavier righthanded neutrinos out of the three that characterise the $v \operatorname{MSM}[5,18]$ or other models, to be almost degenerate in mass. This latter feature is also required for enhanced $\mathrm{CP}$ violation of relevance to leptogenesis [30], which by the way the KR axion field has also an important rôle to play in, as discussed in [13] and mentioned previously, in section 2. In fact, in such scenarios, constant (in cosmic time) backgrounds of the KR form $H_{i j k}$ (spatial components), which violate spontaneously Lorentz and CPT symmetries, may induce lepton asymmetries in the early Universe from the CP and CPT-Violating decays of (sufficiently) heavy sterile neutrinos (distinct from the lightest ones discussed here which play the rôle of DM components). These are interesting issues that deserve further exploration and we hope we shall be able to tackle some of them in the near future.

\section{References}

[1] G. Aad et al. [ATLAS Collaboration], Phys. Lett. B 716, 1 (2012) [arXiv:1207.7214 [hep-ex]]; S. Chatrchyan et al. [CMS Collaboration], Phys. Lett. B 716, 30 (2012) [arXiv:1207.7235 [hep-ex]].

[2] P. Minkowski, Phys. Lett. B 67, 421 (1977); M. Gell-Mann, P. Ramond and R. Slansky, in Supergravity, eds. D.Z. Freedman and P. van Nieuwenhuizen (North-Holland, Amsterdam, 1979); T. Yanagida, in Proc. of the Workshop on the Unified Theory and the Baryon Number in the Universe, Tsukuba, Japan, 1979, eds. O. Sawada and A. Sugamoto; R. N. Mohapatra and G. Senjanović, Phys. Rev. Lett. 44, 912 (1980).

[3] J. Schechter and J. W. F. Valle, Phys. Rev. D 22, 2227 (1980); G. Lazarides, Q. Shafi and C. Wetterich, Nucl. Phys. B 181, 287 (1981); For recent reviews see: R. N. Mohapatra et al., Rept. Prog. Phys. 70, 1757 (2007) [hep-ph/0510213] and references therein.

[4] R. Blumenhagen, M. Cvetic and T. Weigand, Nucl. Phys. B 771, 113 (2007) [hep-th/0609191]; M. Cvetic, R. Richter and T. Weigand, Phys. Rev. D 76, 086002 (2007) [hep-th/0703028].

\footnotetext{
${ }^{1}$ It is important to notice that for exactly three axion species, there is no dependence of the induced right handed neutrino Majorana mass on the effective field theory cut-off in our model [7], but in such multi-axion cases, as discussed previously ( $c f .(2.17)$ ), the axion field has to be superheavy in order for the mass of the sterile neutrino to be at least in the keV range.
} 
[5] T. Asaka, S. Blanchet and M. Shaposhnikov, Phys. Lett. B 631, 151 (2005) doi:10.1016/j.physletb.2005.09.070 [hep-ph/0503065]; M. Shaposhnikov, JHEP 0808, 008 (2008) [arXiv:0804.4542 [hep-ph]] and references therein.

[6] C. R. Argüelles, N. E. Mavromatos, J. A. Rueda and R. Ruffini, JCAP 1604, no. 04, 038 (2016) doi:10.1088/1475-7516/2016/04/038 [arXiv:1502.00136 [astro-ph.GA]]; N. E. Mavromatos, C. R. Argüelles, R. Ruffini and J. A. Rueda, Int. J. Mod. Phys. D 26, no. 03, 1730007 (2016). doi:10.1142/S0218271817300075

[7] N. E. Mavromatos and A. Pilaftsis, Phys. Rev. D 86, 124038 (2012) [arXiv:1209.6387 [hep-ph]].

[8] F. W. Hehl, P. Von Der Heyde, G. D. Kerlick and J. M. Nester, Rev. Mod. Phys. 48, 393 (1976); I. L. Shapiro, Phys. Rept. 357, 113 (2002) [hep-th/0103093] and references therein

[9] M. J. Duncan, N. Kaloper and K. A. Olive, Nucl. Phys. B 387, 215 (1992).

[10] R. Delbourgo and A. Salam, Phys. Lett. B 40, 381 (1972); see, also: S. Weinberg, The Quantum Theory of Fields. Volume II: Modern Applications. (Cambridge University Press 2001) ISBN $0-521-55002-5$.

[11] M. Kalb and P. Ramond, Phys. Rev. D 9, 2273 (1974).

[12] R. R. Metsaev and A. A. Tseytlin, Nucl. Phys. B 293, 385 (1987);

D. J. Gross and J. H. Sloan, Nucl. Phys. B 291, 41 (1987).

[13] N. E. Mavromatos and S. Sarkar, Eur. Phys. J. C 73, no. 3, 2359 (2013) [arXiv:1211.0968 [hep-ph]]. J. Ellis, N. E. Mavromatos and S. Sarkar, Phys. Lett. B 725, 407 (2013) [arXiv:1304.5433 [gr-qc]]. M. de Cesare, N. E. Mavromatos and S. Sarkar, Eur. Phys. J. C 75, no. 10, 514 (2015) doi:10.1140/epjc/s10052-015-3731-z [arXiv:1412.7077 [hep-ph]]. T. Bossingham, N. E. Mavromatos and S. Sarkar, Eur. Phys. J. C 78, no. 2, 113 (2018) doi:10.1140/epjc/s10052-018-5587-5 [arXiv:1712.03312 [hep-ph]].

[14] V. A. Kostelecky and N. Russell, Rev. Mod. Phys. 83, 11 (2011) [arXiv:0801.0287 [hep-ph]].

[15] A. Arvanitaki, S. Dimopoulos, S. Dubovsky, N. Kaloper and J. March-Russell, Phys. Rev. D 81, 123530 (2010) [arXiv:0905.4720 [hep-th]]; M. Cicoli, M. Goodsell and A. Ringwald, arXiv:1206.0819 [hep-th].

[16] J. F. Donoghue, Phys. Rev. D 50, 3874 (1994) [gr-qc/9405057].

[17] T. Asaka, M. Shaposhnikov and A. Kusenko, Phys. Lett. B 638, 401 (2006).

[18] A. Boyarsky, O. Ruchayskiy and M. Shaposhnikov, Ann. Rev. Nucl. Part. Sci. 59, 191 (2009) doi:10.1146/annurev.nucl.010909.083654 [arXiv:0901.0011 [hep-ph]].

[19] R. Ruffini, C. R. Argüelles and J. A. Rueda, Mon. Not. Roy. Astron. Soc. 451, no. 1, 622 (2015) doi:10.1093/mnras/stv1016 [arXiv:1409.7365 [astro-ph.GA]]. See also: I. Siutsou, C. R. Argüelles and R. Ruffini, Astron. Rep. 59, no. 7, 656 (2015) doi:10.1134/S1063772915070124 [arXiv:1402.0695 [astro-ph.GA]]; C. R. Argüelles, R. Ruffini, I. Siutsou and B. Fraga, J. Korean Phys. Soc. 65, no. 6, 801 (2014) doi:10.3938/jkps.65.801 [arXiv:1402.0700 [astro-ph.GA]];

[20] D. Harvey, R. Massey, T. Kitching, A. Taylor, E. Tittley Science 347, 6229, 1462 (2015) and references therein.

[21] J. F. Navarro, C. S. Frenk and S. D. M. White, Astrophys. J. 490, 493 (1997) doi:10.1086/304888 [astro-ph/9611107]. 
[22] B. Moore, Nature 370, 629 - 631 (25 August 1994); doi:10.1038/370629a0; W. J. G. de Blok, The Core-Cusp Problem, Advances in Astronomy 2010789293 (2010) [arXiv:0910.3538]; Se-Heon Oh et al., Astrophys. J. 149 (6), 96 (2015).

[23] B. Moore, S. Ghigna, F. Governato, G. Lake, T. R. Quinn, J. Stadel and P. Tozzi, Astrophys. J. 524, L19 (1999) doi:10.1086/312287 [astro-ph/9907411]; A. A. Klypin, A. V. Kravtsov, O. Valenzuela and F. Prada, Astrophys. J. 522, 82 (1999) doi:10.1086/307643 [astro-ph/9901240]; E. Polisensky and M. Ricotti, Phys. Rev. D 83, 043506 (2011) doi:10.1103/PhysRevD.83.043506 [arXiv:1004.1459 [astro-ph.CO]].

[24] M. Boylan-Kolchin, J. S. Bullock and M. Kaplinghat, Mon. Not. Roy. Astron. Soc. 415, L40 (2011) [arXiv:1103.0007]; ibid. 422, 1203 (2012) [arXiv: 1111.2048].

[25] M. R. Lovell, V. Gonzalez-Perez, S. Bose, A. Boyarsky, S. Cole, C. S. Frenk and O. Ruchayskiy, Mon. Not. Roy. Astron. Soc. 468, no. 3, 2836 (2017) doi:10.1093/mnras/stx621 [arXiv:1611.00005 [astro-ph.GA]].

[26] K. Perez, K. C. Y. Ng, J. F. Beacom, C. Hersh, S. Horiuchi and R. Krivonos, Phys. Rev. D 95, no. 12 , 123002 (2017) doi:10.1103/PhysRevD.95.123002 [arXiv:1609.00667 [astro-ph.HE]].

[27] F. A. Harrison et al., Astrophys. J. 770, 103 (2013) doi:10.1088/0004-637X/770/2/103 [arXiv:1301.7307 [astro-ph.IM]].

[28] J. Diemand, M. Kuhlen, P. Madau, M. Zemp, B. Moore, D. Potter and J. Stadel, Nature 454, 735 (2008) doi:10.1038/nature07153 [arXiv:0805.1244 [astro-ph]]; This is a shallow version of the original profile suggested in: J. Einasto, Trudy Astrofizicheskogo Instituta Alma-Ata 5, 87 (1965); U. Haud and J. Einasto, Astron. Astrophys. 223, 89 (1989).

[29] See for instance: G. K. Leontaris and N. D. Vlachos, Mod. Phys. Lett. A 29, 0179 (2014) [arXiv:1309.5785 [hep-ph]], but with their results applied to the right-handed neutrino sector, and references therein.

[30] For a review see, e.g. A. Pilaftsis, J. Phys. Conf. Ser. 171, 012017 (2009) doi:10.1088/1742-6596/171/1/012017 [arXiv:0904.1182 [hep-ph]], and references therein. 